\title{
FEATURES OF FINDING OPTIMAL SOLUTIONS IN NETWORK PLANNING
}

\author{
Olena Domina \\ «Scientific Route» Ö̈ \\ Narva mnt 7-634, Tallinn, Estonia, Harjumaakond, 10117 \\ info@eu-jr.eu
}

\begin{abstract}
The object of research is a test network diagram, in relation to which the task of minimizing the objective function $q_{\max } / q_{\min } \rightarrow \min$ is posed, which requires maximizing the uniformity of the workload of personnel when implementing an arbitrary project using network planning. The formulation of the optimization problem, therefore, assumed finding such times of the beginning of the execution of operations, taken as input variables, in order to ensure the minimum value of the ratio of the peak workload of personnel to the minimum workload.

The procedure for studying the response surface proposed in the framework of RSM is described in relation to the problem of optimizing network diagrams. A feature of this procedure is the study of the response surface by a combination of two methods - canonical transformation and ridge analysis. This combination of methods for studying the response surface allows to see the difference between optimal solutions in the sense of «extreme» and in the sense of «best». For the considered test network diagram, the results of the canonical transformation showed the position on the response surface of the extrema in the form of maxima, which is unacceptable for the chosen criterion for minimizing the objective function $q_{\max } / q_{\min } \rightarrow \min$. It is shown that the direction of movement towards the best solutions with respect to minimizing the value of the objective function is determined on the basis of a parametric description of the objective function and the restrictions imposed by the experiment planning area. A procedure for constructing nomograms of optimal solutions is proposed, which allows, after its implementation, to purposefully choose the best solutions based on the real network diagrams of your project.

Keywords: Project Network Diagrams, response surface methodology, canonical transformation, ridge analysis, optimal solution nomogram, workload of personnel.
\end{abstract}

DOI: $10.21303 / 2461-4262.2020 .001471$

\section{Introduction}

Optimizing network diagrams is a logical continuation of their construction. However, different end goals of projects give reason to use different approaches to optimization. These approaches are determined by the criteria that are the basis for the selection of target functions. For example, in [1] the following classification of optimization problems was introduced:

- by time, if the duration of work on the diagram is more or less than the prescribed duration;

- by resources, depending on their renewability;

- by cost and resources.

Such a classification, although it is seen as rather capacious, is not exhaustive. A slightly different type of classification is given in [2], according to which it is necessary to distinguish between resource, structural, stochastic optimization methods in network planning. Separation of methods according to this principle is substantiated in this work using a typical triad: «defined variables - objective function - constraints». However, it is quite rightly noted at the same time that any specific practical task has a set of its limitations. In addition to this, it is emphasized that one can't ignore the following circumstances: the model of minimizing the duration of a project with a fixed budget can't always be technically implemented, and a specific problem under consideration may not have solutions under existing constraints. This conclusion, obviously, should be understood in the context of the search for the optimal solutions. That is, one can't ignore the fact that a specific mathematical formulation of the problem must take into account the ultimate goal of the project. It is of interest to see which optimization criteria are used most often. In [3], such a criterion is the cost of the project. The value of the cost change after optimization is introduced in the coordinates «time - cost». A feature of consideration in this work is the use of the concept of additional costs required to accelerate the project. The variant of such a control action should lead, according to the author, to an increase in the efficiency of project implementation. 
A feature of the formulation of the optimization problem in [4] is that it is performed with respect to the least increase in the total cost of work with a reduction in the period of their implementation. This has a certain similarity with the principles outlined in [1]. However, the solution to the problem is presented only in an algorithmic form.

Optimization methods according to the criteria «time», «time - labor resources», «time costs» are considered in [5]. However, the authors limit themselves to conceptual representations of the solution to the problem, focusing not on mathematical delivery and search for optimal solutions, but on the use of classification representations of potentially possible optimization methods.

The work execution time, as the main criterion for optimizing the network diagram, is considered in [6] from the standpoint of a random variable. With this consideration, it is proposed to evaluate not the time of completion of work or the project as a whole, but the probability of the completion date of the project. For this, it is proposed to use the Monte Carlo method, which, in the author's opinion, makes it possible to assess the probability of the completion date of the project for any distribution law of their duration. The probabilistic approach used in this case is practical, but in this work only the principle of finding solutions using Excel is shown outside the mathematical formulation and solution of the problem. It should be noted that the absence of just such a consideration of the problem is a common problem in the study of network diagrams. An exception may be, for example, work [7], in which several variants of the mathematical formulation of the optimization problem are considered. The objective function structure reflects different alternatives and meets the criteria:

- minimizing the cost of the entire project;

- minimizing the risk of critical path operations;

- minimizing the duration of the critical path.

In such options, the optimization of the project plan is carried out under various scenarios formed by constraints of three types:

- for the total cost of the project - when optimizing risk or time);

- for the full duration of the project execution time while optimizing costs or risk;

- by the total amount of risk when optimizing costs or time.

The authors note that the application of the proposed methodology, based on such a variant of the task formalization, reduces the overall risk of the project. However, the paper does not analyze the behavior of the system in the vicinity of optimal solutions. Therefore, it is impossible to evaluate other possible solutions in conditions of different input variables. Such solutions can be obtained using the tools of the response surface methodology (RSM), but for this it is necessary to consider the optimization problem in terms of mathematical experimental design, as was done in [8]. These tools can be used to select the canonical transformation of the response surface and the ridge analysis, the use of which for various applications is described in [9] and [10, 11], respectively. The principles of using this approach to optimize network diagrams, previously not found in the literature, are proposed in this work. It is a continuation of work [8] and aims to select tools for analyzing the response surface to find optimal solutions for choosing the timing of the start of individual operations in the framework of network planning. To achieve this goal, it is necessary to solve the following problems arising directly from the possibility of combining solutions obtained for different alternatives of choosing independent input variables:

- to investigate the behavior of the response surface $q_{\max } / q_{\min }=f\left(x_{1}, x_{2}\right)$ at three points of the D-optimal design for the case when the value of 1 month is taken as the time interval for the shift in the start of operations;

- to investigate the response surface $q_{\max } / q_{\min }=f\left(x_{1}, x_{2}\right)$ at three points of the D-optimal design for the case when the value of 1 quarter is taken for the time interval according to the shift in the start of operations;

- to construct nomograms of optimal solutions describing their behavior depending on the coordinates of the point of the D-optimal design.

\section{Materials and Methods}

To analyze the response surface, let's use the procedure used in [12] to find the optimal solution to the problem of synthesis of a structural material. This procedure allows to establish the behavior of the output variable in the stationary domain by a canonical transformation. After 
that, the answer to the question about the sign-definiteness of the $\mathbf{x}^{\mathrm{T}} \mathbf{A} \mathbf{x}$ quadratic form obtained as a result of this transformation is possible:

- determination of the coordinates of the stationary point $x^{*}$ from the equation:

$$
\left(\begin{array}{ll}
a_{11} & a_{12} \\
a_{21} & a_{22}
\end{array}\right)\left(\begin{array}{l}
x_{1}^{*} \\
x_{2}^{*}
\end{array}\right)=-\left(\begin{array}{l}
a_{1} \\
a_{2}
\end{array}\right),
$$

derived from condition:

$$
\frac{\partial y}{\partial x_{i}}=2 \mathbf{a}+2 A \mathbf{x}^{*}=0
$$

where

$$
y=a_{0}+2 \mathbf{a}^{T} \mathbf{x}+\mathbf{x}^{T} \mathbf{A} \mathbf{x}
$$

and the components of the matrices $\mathbf{a}$ and $\mathbf{A}$ are determined in accordance with [8];

- calculation of the value of the output variable at a stationary point from the equation:

$$
y^{*}=a_{0}+2 \mathbf{a}^{T} \mathbf{x}^{*}+\mathbf{x}^{* T} \mathbf{A} \mathbf{x}^{*},
$$

- obtaining the equation of the response surface in the canonical form:

$$
y-y^{*}=\lambda_{1} \xi_{1}^{2}+\lambda_{2} \xi_{2}^{2},
$$

where $\lambda_{1}, \lambda_{2}-$ the eigenvalues of the matrix $\mathbf{A}[8]$ :

$$
\mathbf{A}=\left(\begin{array}{ll}
1.390335 & 0 \\
0 & -0.27667
\end{array}\right)
$$

- if the value of 1 month is taken as the time interval,

$$
\mathbf{A}=\left(\begin{array}{ll}
-1.99973 & 0.7439 \\
0.7439 & -3.46023
\end{array}\right)
$$

- if the value of 1 quarter is taken as the time interval.

To search for locally optimal solutions, a ridge analysis procedure was used, which allows, in the general case, to analytically describe the lines of final states to find the optimal control $[13,14]$.

In the optimization process, the following network parameters are used:

- early end date of the previous operation $t_{i}^{\prime}$, on a set of previous operations $\omega_{k}^{\prime}$;

- duration of the current operation, $\tau_{k}$;

- early end date of the current operation, $t_{k}^{\prime}$ :

$$
t_{k}^{\prime}=\max \left(t_{i \in \omega_{k}^{\prime}}^{\prime}+\tau_{k}\right),
$$

- late end date of the subsequent operation $t_{j}^{\prime \prime}$, on a set of subsequent operations $\omega_{k}^{\prime \prime}$;

- late end date of the current operation, $t_{k}^{\prime \prime}$ :

$$
t_{k}^{\prime \prime}=\min \left(t_{j \in \omega_{k}^{\prime \prime}}^{\prime \prime}-\tau_{j}\right)
$$

- the length of the directive period:

$$
T=\max _{k} t_{k}^{\prime}
$$

- early start date of the current operation:

$$
t_{k}^{0}=t_{k}^{\prime}-\tau_{k},
$$


Optimization is carried out according to the $q_{\max } / q_{\min } \rightarrow \min$ criterion in the space of normalized input variables, which are used to select the times of the beginning of operations $C$ (for alternative 1) and $Q+P$ (for alternative 2) - Table 1 [8].

Table 1

Choice of input variables and variation intervals for the optimized network diagram

\begin{tabular}{|c|c|c|c|c|c|c|c|c|c|c|c|}
\hline Work identifier & $q_{k}$ & $\tau_{k}$ & $\omega_{k}^{\prime}$ & $\omega_{k}^{\prime \prime}$ & $t_{k}^{\prime}$ & $t_{k}^{\prime \prime}$ & $\Delta \tau$ & $t_{k}^{0}$ & $t_{k}^{0}(\mathbf{m i n})$ & $t_{k}^{0}(\mathbf{m a x})$ & $t_{k}^{0} \mathbf{( 0 )}$ \\
\hline$D$ & 20 & 1 & - & $B, N, F$ & 1 & 2 & 1 & 0 & & & \\
\hline$C$ & 20 & 1 & - & $B, H, K$ & 1 & 5 & 4 & $\mathbf{0}$ & $\mathbf{0}$ & 4 & 2 \\
\hline$E$ & 4 & 2 & - & $F$ & 2 & 2 & 0 & 0 & & & \\
\hline$A$ & 8 & 1 & - & $B, N$ & 1 & 5 & 4 & 0 & & & \\
\hline$F$ & 16 & 3 & $D, E$ & $G, P$ & 5 & 5 & 0 & 2 & & & \\
\hline$H$ & 4 & 1 & $C$ & $L, M$ & 2 & 12 & 10 & 1 & & & \\
\hline$Q$ & 10 & 6 & - & $\boldsymbol{R}$ & 6 & 11 & 5 & $\mathbf{0}$ & $\mathbf{0}$ & 4 & 2 \\
\hline$N$ & 1 & 4 & $A, D$ & $I$ & 5 & 9 & 4 & 1 & & & \\
\hline$G$ & 5 & 4 & $F$ & $I$ & 9 & 9 & 0 & 5 & & & \\
\hline$B$ & 13 & 4 & $A, C, D$ & $I$ & 5 & 9 & 4 & 1 & & & \\
\hline$R$ & 7 & 3 & $Q$ & - & 9 & 14 & 5 & 6 & & & \\
\hline$P$ & 10 & 2 & $F$ & - & 7 & 14 & 7 & 5 & 5 & 11 & 8 \\
\hline$M$ & 2 & 2 & $H$ & - & 4 & 14 & 10 & 2 & & & \\
\hline$L$ & 6 & 2 & $H$ & - & 4 & 14 & 10 & 2 & & & \\
\hline$K$ & 2 & 2 & $C$ & - & 3 & 14 & 11 & 1 & & & \\
\hline$I$ & 3 & 5 & $B, G, N$ & - & 14 & 14 & 0 & 9 & & & \\
\hline
\end{tabular}

In Table 1, the following color highlights are used: red-operation of alternative No. 1, pink-operation of alternative No. 2

The normalization, which transmit the values of the input variables into the dimensionless range $[-1 ;+1]$, is carried out according to the formula:

$$
x_{\text {inorm }}=\frac{2 x_{i}-\left(x_{i \max }+x_{i \min }\right)}{x_{i \max }-x_{i \min }}, i=1,2, \ldots, N, j=1,2, \ldots, n,
$$

where $x_{i \max }$ - the maximum value of the $i$-th input variable in the selected range of variation in natural form; $x_{i \min }$ - the minimum value of the $i$-th input variable in the selected range of variation in natural form; $x_{\text {inorm }}$ - the value of the $i$-th input variable in normalized form; $x_{i}$ - the value of the $i$-th input variable in natural form ( $i=1,2$ for alternative No. 2, $i=3$ for alternative No. 1$)$.

Taking as a generalized designation of the input variable $t_{k}^{0}(k \rightarrow Q, P$ for alternative No. 2, $k \rightarrow C$, for alternative No. 1), the variation intervals are presented in the form of the following correspondences:

$x_{1} \rightarrow\left\{\begin{array}{l}t_{Q}^{0}(\min ), \\ t_{Q}^{0}(\max ),\end{array}-\right.$ for alternative No. 2;

$x_{2} \rightarrow\left\{\begin{array}{l}t_{P}^{0}(\min ), \\ t_{P}^{0}(\max ),\end{array}-\right.$ for alternative No. $2 ;$

$x_{3} \rightarrow\left\{\begin{array}{l}t_{C}^{0}(\min ), \\ t_{C}^{0}(\max ),\end{array}-\right.$ for alternative No. $1 ;$

$t_{k}^{0}(\min )$ - the lower value of the range of variation of the input variable, which is the moment when the current operation starts;

$t_{k}^{0}(\max )$ - the upper value of the range of variation of the input variable, which is the moment when the current operation starts. 
Taking into account the accepted designations, the boundaries of the variation intervals are as follows:

- for alternative No. 2:

$$
\begin{aligned}
& x_{1}^{\min }=t_{Q}^{0}(\min )=t_{Q}^{\prime}-\tau_{Q}=0, \\
& x_{1}^{\max }=t_{Q}^{0}(\max )=t_{Q}^{\prime \prime}-\tau_{Q}=4, \\
& x_{2}^{\min }=t_{P}^{0}(\min )=t_{P}^{\prime}-\tau_{P}=5, \\
& x_{2}^{\max }=t_{P}^{0}(\max )=t_{P}^{\prime \prime}-\tau_{P}=11 ;
\end{aligned}
$$

- for alternative No. 1:

$$
\begin{aligned}
& x_{3}^{\min }=t_{C}^{0}(\min )=t_{C}^{\prime}-\tau_{C}=0, \\
& x_{3}^{\max }=t_{C}^{0}(\max )=t_{C}^{\prime \prime}-\tau_{C}=4 .
\end{aligned}
$$

\section{Experimental procedures}

Numerical modeling is carried out based on the canonical transformation of the response surface described by the regression equations [8]:

- if the value of 1 month is taken as the time interval for the offset of the start of operations:

$$
y=18.44433-1.66733 x_{1 \text { norm }}+1.390335 x_{1 \text { norm }}^{2}-0.27667 x_{2 \text { norm }}^{2} ;
$$

- if the value of 1 quarter is taken as the time interval for the offset of the start of operations:

$$
\begin{gathered}
y=9.84867-4.1555 x_{1 \text { norm }}-3.31316 x_{2 \text { norm }}-1.99973 x_{1 \text { norm }}^{2}-3.46023 x_{2 \text { norm }}^{2}- \\
-0.27667 x_{2 \text { norm }}^{2}+1.48775 x_{1 \text { norm }} x_{2 \text { norm }} .
\end{gathered}
$$

To test the possibility of obtaining a better result than the result provided by alternative 2 , optimization is carried out at the points of the D-optimal design corresponding to the implementation of alternative 1 [8].

\section{Results}

4. 1. Results for the calculation interval of the output variable 1 month

The equation of the response surface in the canonical form for the case when the value of 1 month is taken for the time interval according to the displacement of the start of operations is as follows:

$$
y-y^{*}=-0.27667 \xi_{1}^{2}+1.390335 \xi_{2}^{2},
$$

where $\left(\xi_{1} ; \xi_{2}\right)$ - a new coordinate system obtained from the old system $\left(x_{1 \text { norm }} ; x_{2 n o r m}\right)$ by translating and rotating the axes.

Taking into account the value of the output variable at the stationary point, the canonical form of the response surface takes the form:

$$
y-17.202=-0.27667 \xi_{1}^{2}+1.390335 \xi_{2}^{2} .
$$

From (20) it is seen that the eigenvalues have different signs. Therefore, the quadratic form $L\left(\xi_{1}, \xi_{2}\right)$ is alternating. By nature, based on the fact that $\left|\lambda_{1}\right| \neq\left|\lambda_{2}\right|, \lambda_{1}<0, \lambda_{2}>0$ the response surface has a saddle point. This can be seen by visualization obtained by direct construction of the response surface according to equation (17) - Fig. 1. 


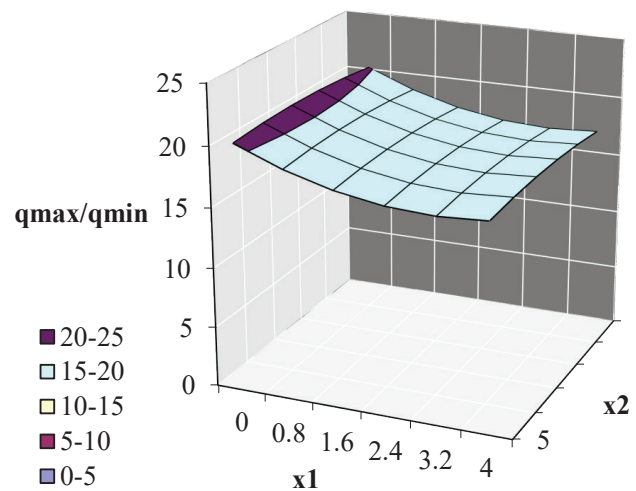

Fig. 1. Response surface for the case when for the time interval according to the displacement of the beginning of operations to take the value of 1 month for point D-optimal design No. $1\left(x_{3}=-1\right)$

Table 2 shows the results of calculating the parameters of the regression equations for points $2\left(x_{3}=0\right)$ and $3\left(x_{3}=+1\right)$ of the D-optimal design of alternative 1 .

Table 2

The results of calculating the coefficients of the regression equations for points No. 2 and No. 3 of the D-optimal design of alternative 1

\begin{tabular}{|c|c|c|c|c|c|}
\hline $\begin{array}{c}\text { D-optimal } \\
\text { design point }\end{array}$ & $a_{0}$ & $\left(\begin{array}{l}a_{1} \\
a_{2}\end{array}\right)$ & $\mathbf{A}$ & $\lambda_{1}$ & $\lambda_{2}$ \\
\hline No. 2 & 17.70331 & $\left(\begin{array}{l}-0.305 \\
-0.417\end{array}\right)$ & $\left(\begin{array}{ll}-0.9720 & -0.2084 \\
-0.2084 & -0.6385\end{array}\right)$ & -1.0721 & -0.53829 \\
\hline No. 3 & 16.21134 & $\left(\begin{array}{l}-0.2500 \\
-0.1944\end{array}\right)$ & $\left(\begin{array}{ll}0.2582 & 0.1666 \\
0.1666 & -0.4083\end{array}\right)$ & -0.44766 & 0.297489 \\
\hline
\end{tabular}

The response surface equation for point D-optimal design No. $2\left(x_{3}=0\right)$ :

$$
y-y^{*}=-1.0721 \xi_{1}^{2}-0.53829 \xi_{2}^{2}
$$

Taking into account the value of the output variable at the stationary point, the canonical form of the response surface takes the form:

$$
y-18.007=-1.0721 \xi_{1}^{2}-0.53829 \xi_{2}^{2} .
$$

From (22) it is seen that the eigenvalues have the same negative signs. Therefore, the quadratic form $L\left(\xi_{1}, \xi_{2}\right)$ is negative definite. The response surface has a maximum point, which can be seen by visualization obtained by direct construction of the response surface based on the parameters of the regression equation given in Table 2 for point No. 2 of the D-optimal design of alternative 1 (Fig. 2).

The response surface equation for point D-optimal design No. $3\left(x_{3}=0\right)$ :

$$
y-y^{*}=-0.4477 \xi_{1}^{2}+0.2975 \xi_{2}^{2}
$$

Taking into account the value of the output variable at the stationary point, the canonical form of the response surface takes the form:

$$
y-15.9714=-0.4477 \xi_{1}^{2}+0.2975 \xi_{2}^{2} .
$$


It can be seen from (24) that the eigenvalues have different signs. Therefore, the quadratic form is alternating. Since $\left|\lambda_{1}\right| \neq\left|\lambda_{2}\right|, \lambda_{1}<0, \lambda_{2}>0$ the response surface has a saddle point. This can be seen on the response surface, built on the basis of the parameters of the regression equation shown in Table 2 for point No. 3 of the D-optimal design of alternative 1 (Fig. 3).

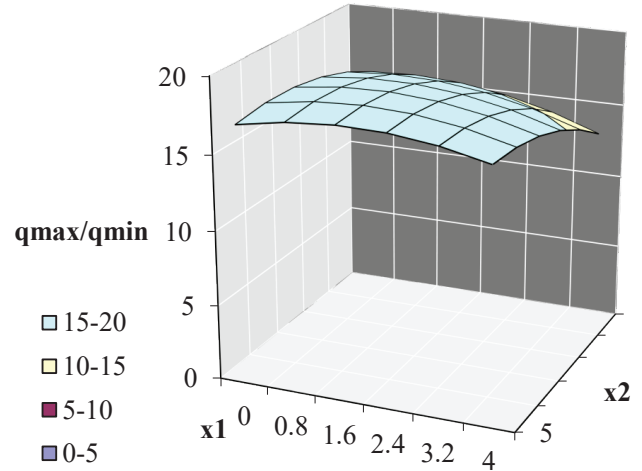

Fig. 2. The response surface for the case when for the time interval according to the displacement of the beginning of operations to take the value of 1 month for the point of the D-optimal design No. $2\left(x_{3}=0\right)$

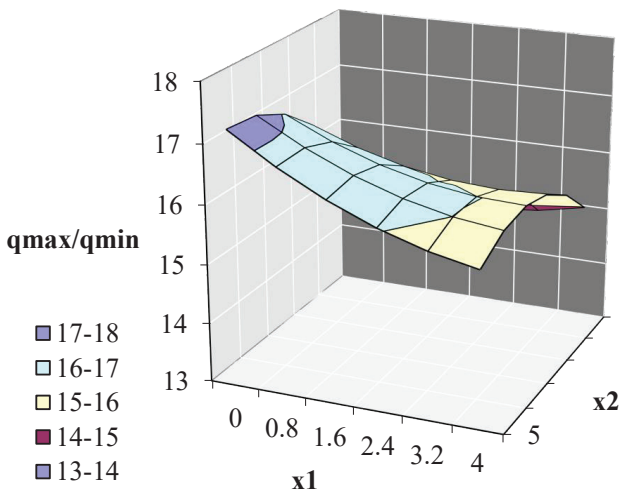

Fig. 3. The response surface for the case when for the time interval according to the displacement of the beginning of operations to take the value of 1 month for the point of the D-optimal design No. $3\left(x_{1}=+1\right)$

\section{2. Results for the interval of calculation of the output variable 1 quarter}

An equation for the response surface is obtained in canonical form for the case when the value of 1 quarter is taken as the time interval for the displacement of the start of operations:

$$
\begin{gathered}
y-y^{*}=-3.77241 \xi_{1}^{2}-1.68755 \xi_{2}^{2}, \\
y-17.4885=-3.77241 \xi_{1}^{2}-1.68755 \xi_{2}^{2} .
\end{gathered}
$$

It is seen from (11) that the eigenvalues have the same negative signs, and, taking into account that $\left|\lambda_{1}\right| \neq\left|\lambda_{2}\right|, \lambda_{1}<0, \lambda_{2}<0$, the quadratic form $L\left(\xi_{1}, \xi_{2}\right)$ is negative definite. The response surface has a maximum point, which can also be seen by visualization obtained by direct construction of the response surface according to equation (18) - Fig. 4.

For the problem being solved, requiring minimization of the $q_{\max } / q_{\min }$ value, the best solution is not the values of the input variables at the extreme point, which has the character of a maximum, but the value at the boundary of the planning area corresponding to the point with coordinates $(4 ; 11)$. That is, in this case the condition $x_{1} \rightarrow \max , x_{2} \rightarrow \max$ must be satisfied. Only at such a point $q_{\max } / q_{\min } \rightarrow \min$, since there is a distance from the ellipsoid apex (Fig. 5). Fig. 5 shows the level lines, and the area of the best solutions corresponds to blue. 


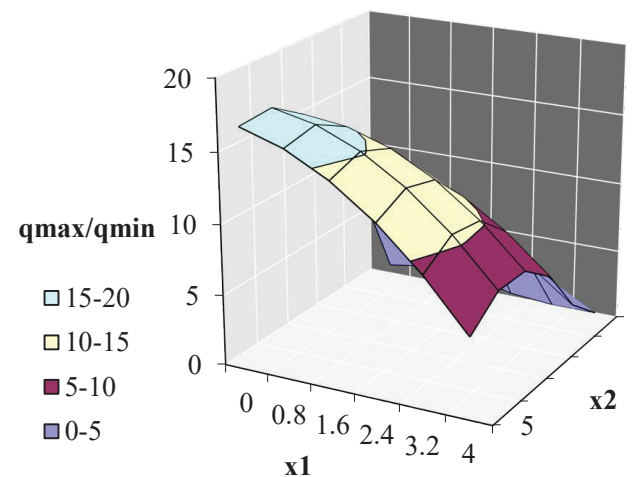

Fig. 4. The response surface for the case when the value of 1 quarter is taken for the time interval according to the displacement of the start of operations

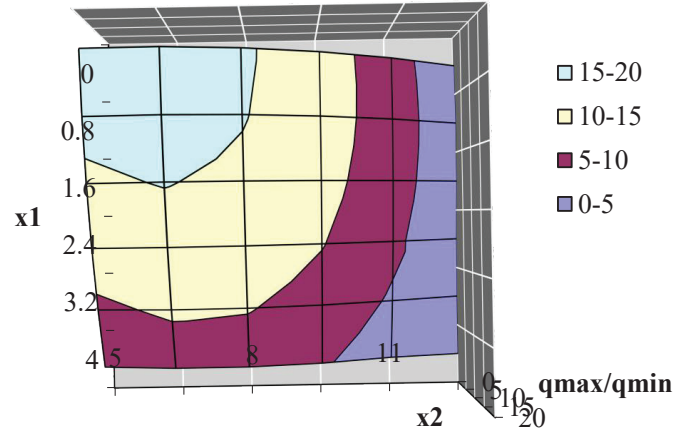

Fig. 5. Level lines near the range of values of the output variable for the case when for the time interval according to the displacement of the start of operations to take the value of 1 quarter for the point of the D-optimal design No. $1\left(x_{3}=-1\right)$

Table 3 shows the results of calculating the parameters of the regression equations for points $2\left(x_{3}=0\right)$ and $3\left(x_{3}=+1\right)$ of the D-optimal design of alternative 1 , when the value of 1 quarter is taken as the time interval for the shift in the start of operations.

\section{Table 3}

The results of calculating the coefficients of the regression equations for points No. 2 and No. 3 of the D-optimal design of alternative 1

\begin{tabular}{|c|c|c|c|c|c|}
\hline $\begin{array}{c}\text { D-optimal } \\
\text { design point }\end{array}$ & $a_{0}$ & $\left(\begin{array}{l}a_{1} \\
a_{2}\end{array}\right)$ & $\mathbf{A}$ & $\lambda_{1}$ & $\lambda_{2}$ \\
\hline No. 2 & 14.23766 & $\left(\begin{array}{l}-1.7974 \\
-2.0064\end{array}\right)$ & $\left(\begin{array}{cc}-3.4096 & 0.7596 \\
0.7596 & -2.7876\end{array}\right)$ & -3.91939 & -2.27779 \\
\hline No. 3 & 11.87818 & $\left(\begin{array}{l}-1.2238 \\
-1.4301\end{array}\right)$ & $\left(\begin{array}{lr}-2.7673 & 0.3716 \\
0.3716 & -2.8338\end{array}\right)$ & -3.17366 & -2.42749 \\
\hline
\end{tabular}

The response surface equation for point D-optimal design No. $2\left(x_{3}=0\right)$ :

$$
y-y^{*}=-3.9194 \xi_{1}^{2}-2.2778 \xi_{2}^{2}
$$

Taking into account the value of the output variable at the stationary point, the canonical form of the response surface takes the form:

$$
y-17.3975=-3.9194 \xi_{1}^{2}-2.2778 \xi_{2}^{2} .
$$


It is seen from (28) that the eigenvalues have the same negative signs. Therefore, the quadratic form $L\left(\xi_{1}, \xi_{2}\right)$ is negative definite. The response surface has a maximum point, which can be seen by visualization obtained by direct construction of the response surface based on the parameters of the regression equation shown in Table 3 for point No. 2 of the D-optimal design of alternative 1 (Fig. 6).

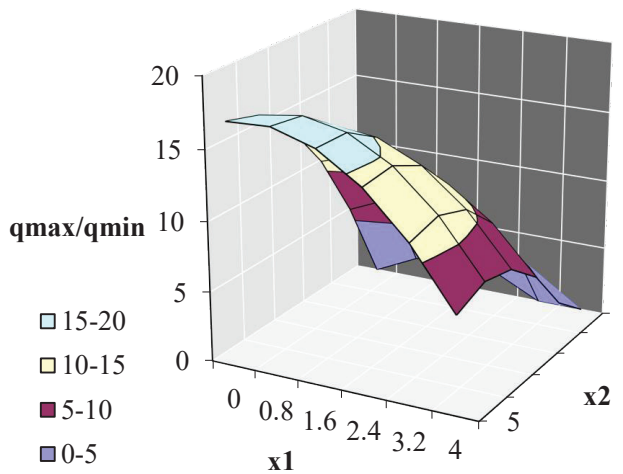

Fig. 6. The response surface for the case when for the time interval according

to the displacement of the beginning of operations to take the value of 1 quarter for the point of the D-optimal design No. $2\left(x_{3}=0\right)$

The response surface equation for point D-optimal design No. $3\left(x_{3}=0\right)$ :

$$
y-y^{*}=-3.17366 \xi_{1}^{2}-2.42749 \xi_{2}^{2} .
$$

Taking into account the value of the output variable at the stationary point, the canonical form of the response surface takes the form:

$$
y-13.3326=-3.17366 \xi_{1}^{2}-2.42749 \xi_{2}^{2} .
$$

It can be seen from (30) that the eigenvalues have the same negative signs. Therefore, the quadratic form $L\left(\xi_{1}, \xi_{2}\right)$ is negative definite. The response surface has a maximum point, which can be seen by visualization obtained by direct construction of the response surface based on the parameters of the regression equation shown in Table 3 for point No. 3 of the D-optimal design of alternative 1 (Fig. 7).

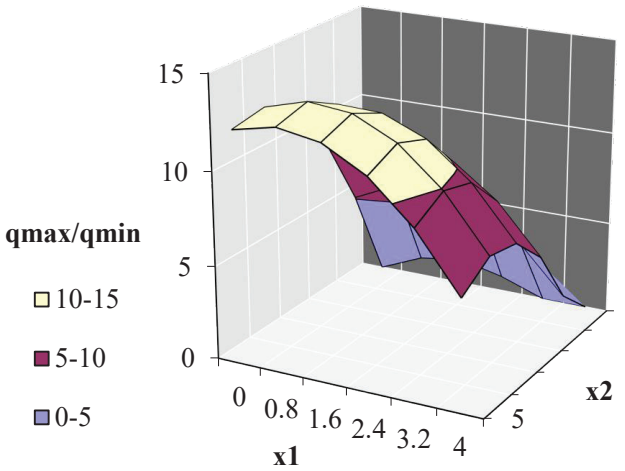

Fig. 7. Response surface for the case when the value of 1 quarter for the point of D-optimal design No. $2\left(x_{3}=+1\right)$

\section{3. Optimal solution nomograms}

Optimal solutions with respect to the early timing of operations that satisfy the conditions for the input variables introduced in [8] are obtained in a parametric form. Therefore, a convenient 
variant of their representation can be nomograms, examples of the construction of which in the case of a parametric description of the optimization problem are given in [15-17]. In the case under consideration, these nomograms are a parametric form of a family of dependencies of the type $x_{1}(\lambda)=f\left(x_{2}(\lambda)\right)$, in which $\lambda$ acts as a parameter, the domains of which are formed based on the position of the poles for the ridge lines. The components of the vector of input variables are determined in this case by solving the equation:

$$
\mathbf{x}^{*}(\lambda)=(\lambda \mathbf{I}-\mathbf{A})^{-1} \mathbf{a},
$$

where the components of the matrices $\mathbf{A}$ and $\mathbf{a}$ are selected from Tables 2, 3 for points No. 2 and No. 3 of the D-optimal design. The components of the matrices $\mathbf{A}$ and $\mathbf{a}$ for point No. 1 of the D-optimal design are given in [8].

Fig. 8, 9 show such nomograms, built based on the results of the ridge analysis for point No. $1\left(x_{3}=-1\right)$ ) of the D-optimal design of alternative 1 in the case when the time interval for the shift in the start of operations is taken as 1 month and 1 quarter, respectively. The nomograms are built in a normalized form; for ease of perception, they are denoted in the Fig. 8, 9 by $x_{1}$ and $x_{2}$.

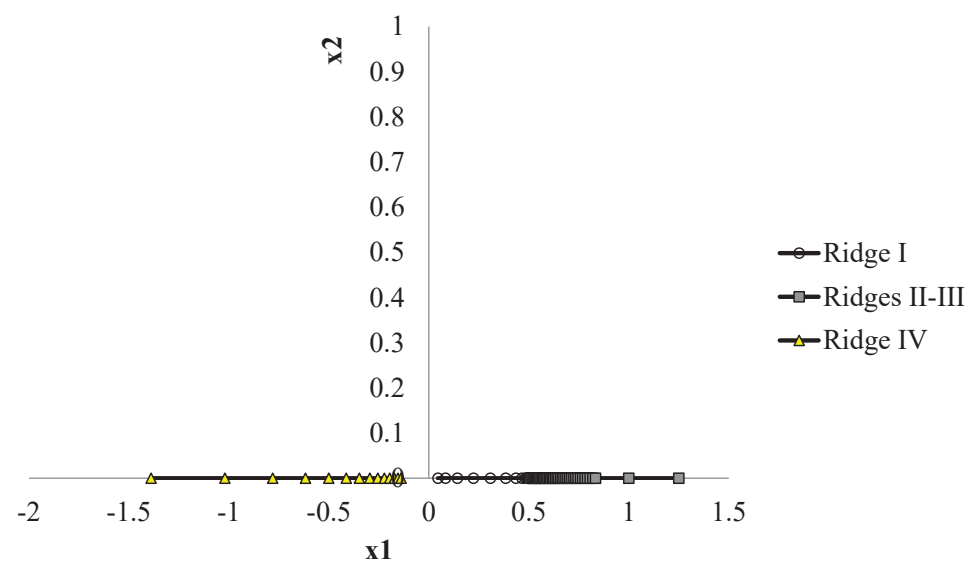

Fig. 8. Nomogram of optimal solutions for point No. $1\left(x_{3}=-1\right)$ of the D-optimal design of alternative 1 in the case when the value of 1 month is taken for the time interval according to the shift in the start of operations

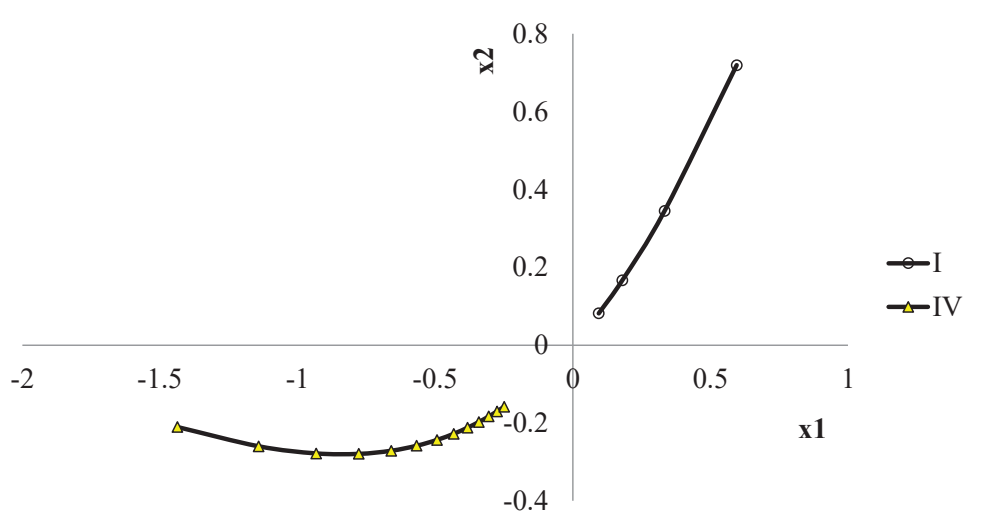

Fig. 9. Nomogram of optimal solutions for point No. $1\left(x_{3}=-1\right)$ of the D-optimal design of alternative 1 in the case when the value of 1 quarter is taken over the time interval by the shift in the start of operations

Fig. 10, 11 show nomograms constructed based on the results of the ridge analysis for point No. $2\left(x_{3}=0\right)$ of the D-optimal design of alternative 1 in the case when the value of 1 month and 1 quarter, respectively, is taken as the time interval for the shift in the start of operations. 


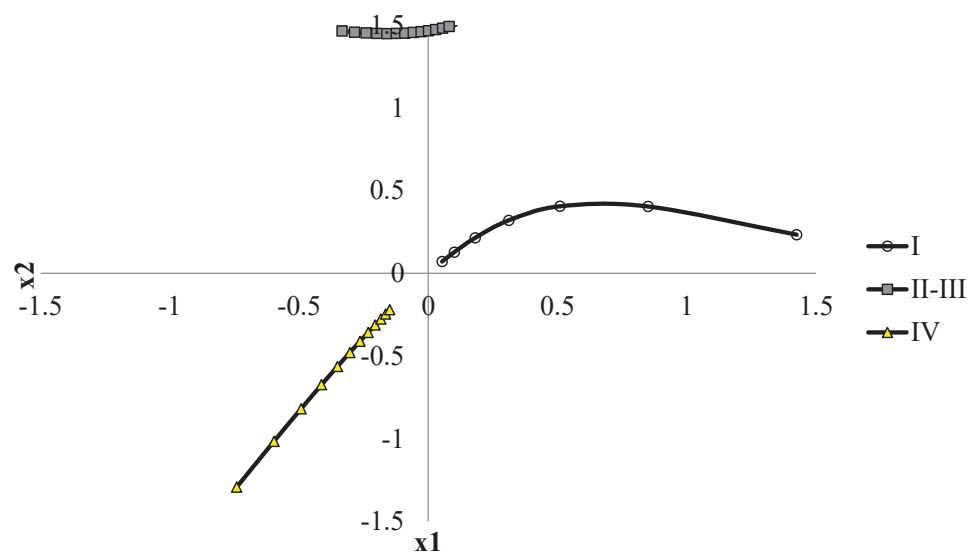

Fig. 10. Nomogram of optimal solutions for point No. $2\left(x_{3}=0\right)$ of the D-optimal design of alternative 1 in the case when the value of 1 month is taken for the time interval according to the shift in the start of operations

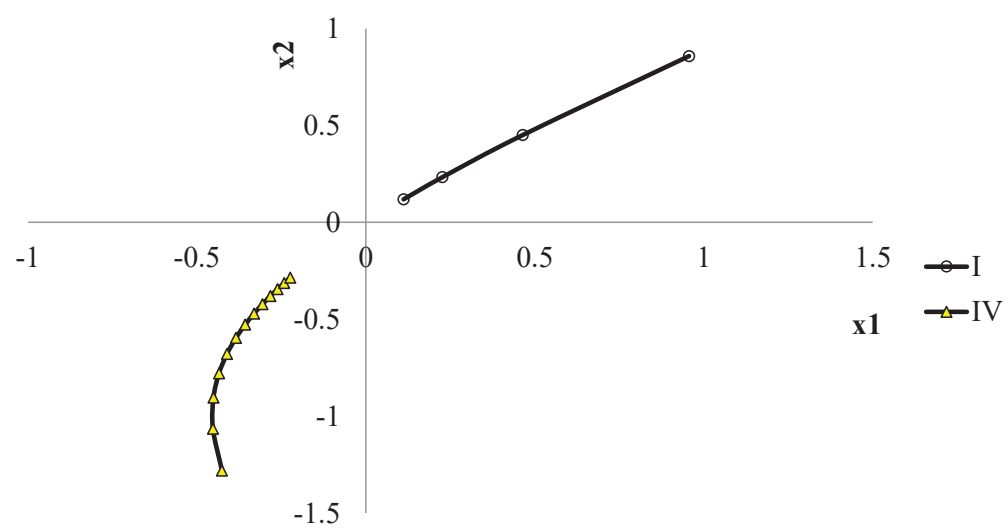

Fig. 11. Nomogram of optimal solutions for point No. $2\left(x_{3}=0\right)$ of the D-optimal design of alternative 1 in the case when the value of 1 quarter is taken over the time interval according to the shift in the start of operations

Fig. 12, 13 show nomograms based on the results of the ridge analysis for point No. $3\left(x_{3}=+1\right)$ of the D-optimal design of alternative 1 in the case when the value of 1 month and 1 quarter, respectively, is taken as the time interval for the offset of the start of operations.

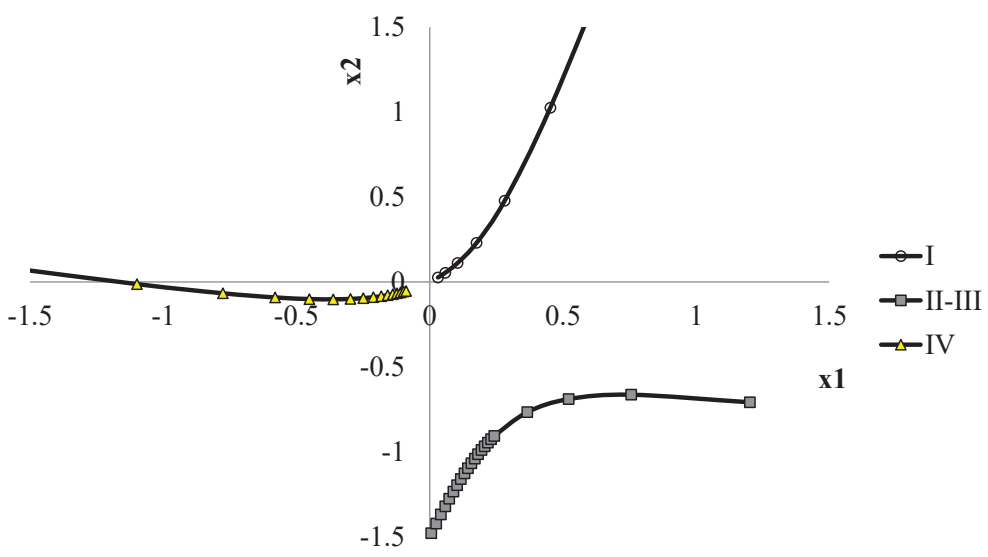

Fig. 12. Nomogram of optimal solutions for point No. $3\left(x_{3}=+1\right)$ of the D-optimal design of alternative 1 in the case when the value of 1 month is taken for the time interval according to the shift in the start of operations 


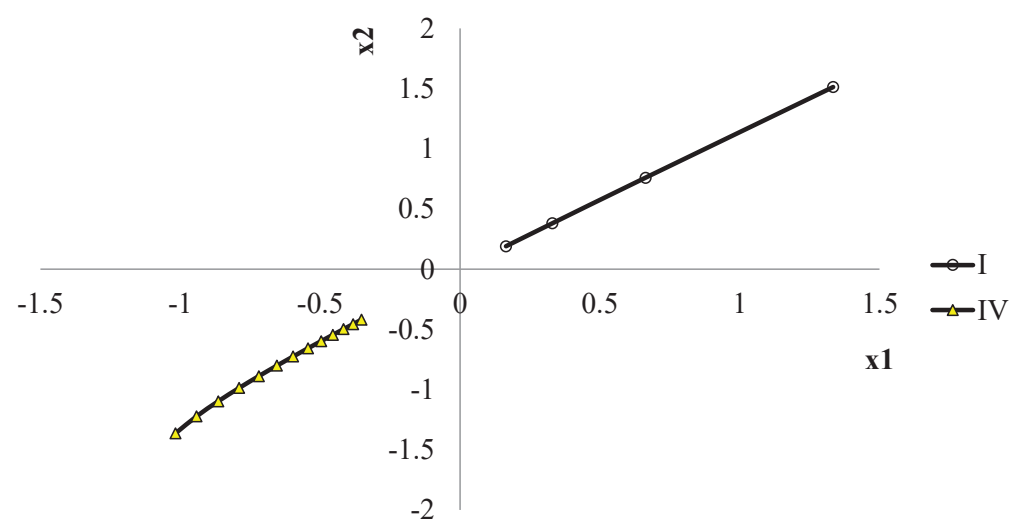

Fig. 13. Nomogram of optimal solutions for point No. $3\left(x_{3}=+1\right)$ of the D-optimal design of alternative 1 in the case when the value of 1 quarter is taken over the time interval by the shift in the start of operations

Fig. 14, 15 show the generalized form of nomograms of optimal solutions for cases when the time interval for the shift in the start of operations is taken as 1 month and 1 quarter, respectively.

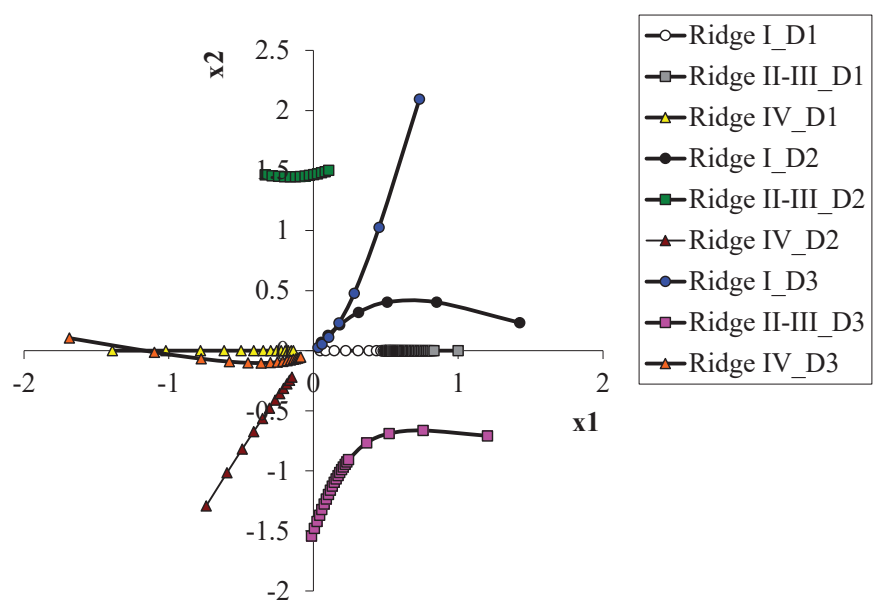

Fig. 14. Nomogram of optimal solutions for the case when for the time interval for the shift in the beginning of operations to take the value of 1 month
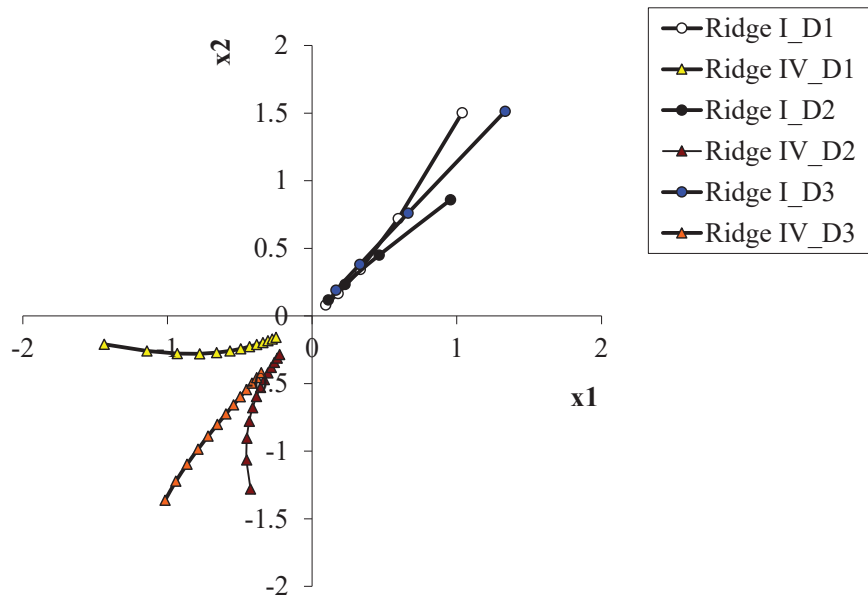

Fig. 15. Nomogram of optimal solutions for the case when for the time interval according to the shift in the beginning of operations to take the value of 1 quarter 
Shown in Fig. 14, 15 nomograms allow finding optimal solutions that satisfy the criterion $q_{\max } / q_{\min } \rightarrow \min$ in the space of normalized input variables at different distances from the center of the experimental design.

\section{Discussion}

The coordinates of points on the lines of the nomograms of optimal solutions represent the points of intersection of the response surface and the surface of constraints imposed on the input variables by the real parameters of the network diagram. The latter are formed directly by the amount of time reserves for performing operations that meet the following conditions [8]:

- intensity of such operations is maximum;

- the number of immediately preceding operations is minimal;

- time reserve for the operation is sufficient for variation.

Perhaps let's expect that with respect to the variable $x_{2}$, the best result from the point of view of achieving the optimization criterion $q_{\max } / q_{\min } \rightarrow \min$ will be for $x_{2}=12$. This is permissible taking into account the fact that the upper admissible value of the late start date of the operation $P$ is $t_{P}^{0}(\max )=12$ (Table 1$)$, and the value $t_{P}^{0}(\max )=11$ is chosen in [8] from the consideration of operating with integers with respect to points in time. Consequently, the optimal network diagram will have the form in which the beginning of the operation $\mathrm{P}$ is shifted to the moment of time 12, which will provide:

- value $q_{\max } / q_{\min }=4.7$ in the case when the value of 1 month $\left(q_{\max }=47\right.$ for the interval $4-5$ and $q_{\min }=10$ for the interval $10-11$ );

- the value $q_{\max } / q_{\min }=2.37$ in the case when the value of 1 quarter $\left(q_{\max }=34\right.$ for quarter 3 and $q_{\min }=14.33$ for quarter 4$)$ is taken as the time interval for the offset of the start of operations.

In this case, the area of limitations, outlined by the radius $r=\left(x_{1}^{2}+x_{2}^{2}\right)^{1 / 2}$, can be expanded from 1.41 to 1.67 , which will allow taking readings from the nomograms of optimal solutions in a wider range (Fig. 14, 15).

The optimal network diagram in this case will be as shown in Fig. 16. The following color designations are used: pink color - actions on operations taken as independent input variables, orange color - actions on operations corresponding to dependent input variables.

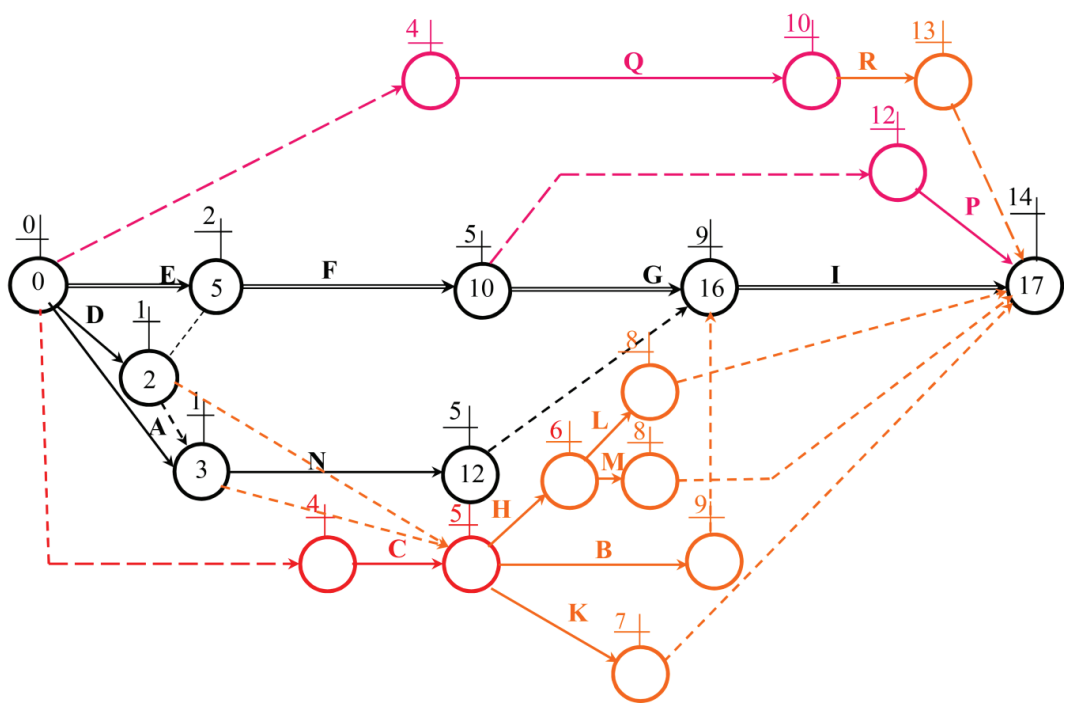

Fig. 16. Optimal network diagram

It should be noted that the application of the response surface analysis procedure, which combines the canonical transformation and the ridge analysis, makes it possible to establish the best solutions in terms of achieving a given requirement for the value of the objective function. This allows to see the difference between the term «optimal» in the sense of «extreme» and «optimal» in the sense of «best for a given objective function». If the first is described by the results of 
the canonical transformation, then the second is described by the ridge analysis. In the considered example, where the objective function requires minimization, the optimal solutions are found precisely from the results of the ridge analysis, and these solutions are obtained when moving along the response surface from extreme values. Using nomograms of optimal solutions (Fig. 14, 15) allows to see all possible optimal solutions in the area limited by the experimental design. It is important to note that such restrictions are strict, since they are regulated by time reserves, so it is not possible to extrapolate them outside this area.

A limitation of this study is the impossibility to apply the ready-made solutions obtained for an arbitrary network diagram. It is possible only apply the entire procedure, the ultimate goal of which is to obtain optimal solutions for your network diagram. A possible continuation of this study could be the study of the question of how adequately the proposed procedure can be used when choosing other optimization criteria, or, in general, other target functions.

The solutions obtained in this case can form the initial data for the implementation of the algorithm for choosing winning strategies [18], which will take into account the cost criteria in addition to the selected optimization criterion in the form of uniformity of staff load.

\section{Conclusions}

The procedure for studying the response surface proposed in the framework of RSM in relation to the problem of optimizing network diagrams allows to see solutions that meet the specified criteria. The essence of this procedure consists in converting the analytical description of the response surface to the canonical form and subsequent ridge analysis. This combination of methods for studying the response surface allows to see the difference between optimal solutions in the sense of «extreme» and in the sense of «best for a given objective function». So, for the considered test network diagram, in relation to which the minimization of the objective function was required, the results of the canonical transformation showed the position on some response surfaces of the extrema in the form of maxima. That is, to achieve the best solutions, it is required to move from these points. It is shown that the direction of such a movement, the purpose of which is to minimize the value of the objective function, can be determined on the basis of a parametric description of the objective function and the constraints imposed by the experiment planning domain. Moreover, such restrictions are strict, since they are regulated by the time reserves for performing those operations that are selected as independent input variables.

The application of the proposed procedure is illustrated by two examples in relation to the test network schedule: for the case when the value of 1 month is taken for the time interval according to the displacement of the start of operations, and for the case when the value of 1 quarter is taken as the time interval for the displacement of the start of operations. Obtaining optimal solutions for such different options allows to additionally solve the problem with respect to which time interval is better to choose to optimize the distribution of labor resources. In the examples considered, such the best interval turned out to be 1 quarter, since in this case, the value of the objective function was reduced by almost $50 \%$ - from $q_{\max } / q_{\min }=4.7$ to $q_{\max } / q_{\min }=2.37$.

The use of the proposed nomograms of optimal solutions allows to purposefully select the best solutions based on your real network schedules. Therefore, despite the fact that the results obtained can't be explicitly transferred to any network schedule, the proposed procedure can be applied to an arbitrary schedule when planning projects.

\section{References}

[1] Karenov, R. S. (2013). Methods of analysis and optimization of network schedule. Vestnik Karagandinskogo universiteta. Seriya «Matematika», 3 (71), 53-65.

[2] Kushner, M. A. (2010). Minimization model of project due dates in the form of network technologies and a fixed budget. Vestnik Astrahanskogo gosudarstvennogo tehnicheskogo universiteta. Seriya: Ekonomika, 2, 124-129.

[3] Shorikov, A. F., Butsenko, Y. V. (2015). Investment planning optimization methods based on network modeling and their applications. Vestnik Permskogo universiteta. Seriya: Ekonomik, 4 (27), 62-70.

[4] Tihobaev, V. M., Tolokonnikov, L. A., Shatohina, A. G. (2010). Optimization of complex plan works in interchangeable resources using the network graph. Izvestiya Tul'skogo gosudarstvennogo universiteta. Ekonomicheskie i yuridicheskie nauki, 2-2, 143-150. 
[5] Gorbaneva, E. P., Ovchinnikova, E. V., Sevryukova, K. S. (2018). Optimization of the Network Schedule in Conditions of Limited Resources. Safety of critical infrastructures and territories. Safety Problems of Civil Engineering Critical Infrastructures - Safety2018. Ekaterinburg, 143-151.

[6] Katargin, N. V. (2012). Optimization of the network schedule of a complex of operations. Upravlencheskie nauki, 1, 87-93.

[7] Shmat, V. V., Yuva, D. S. (2017). Methodology of risk-optimizing planning development for the innovative project in the oil and gas sector. Innovatsii, 6 (224), 113-121

[8] Domina, O. (2020). Selection of alternative solutions in the optimization problem of network diagrams of project implementation. Technology Audit and Production Reserves, 4 (4 (54)), 9-22. doi: https://doi.org/10.15587/2706-5448.2020.210848

[9] Akimov, O., Penzev, P., Marynenko, D., Saltykov, L. (2018). Identification of the behavior of properties of a cold-hardening glass-liquid mixture with propylene-carbonate different in dosing components. Technology Audit and Production Reserves, 2 (3 (46)), 4-9. doi: https://doi.org/10.15587/2312-8372.2019.169748

[10] Dotsenko, Y., Dotsenko, N., Tkachyna, Y., Fedorenko, V., Tsybulskyi, Y. (2018). Operation optimization of holding furnaces in special casting shops. Technology Audit and Production Reserves, 6 (1 (44)), 18-22. doi: https://doi.org/10.15587/ 2312-8372.2018.150585

[11] Chibichik, O., Sil'chenko, K., Zemliachenko, D., Korchaka, I., Makarenko, D. (2017). Investigation of the response surface describing the mathematical model of the effects of the $\mathrm{Al} / \mathrm{Mg}$ rate and temperature on the Al-Mg alloy castability. ScienceRise, 5 (2), 42-45. doi: https://doi.org/10.15587/2313-8416.2017.101923

[12] Demin, D. (2017). Strength analysis of lamellar graphite cast iron in the «carbon (C) - carbon equivalent (Ceq)» factor space in the range of $\mathrm{C}=(3.425-3.563) \%$ and $\mathrm{Ceq}=(4.214-4.372) \%$. Technology Audit and Production Reserves, 1 (1 (33)), 24-32. doi: https://doi.org/10.15587/2312-8372.2017.93178

[13] Demin, D. (2017). Synthesis of optimal control of technological processes based on a multialternative parametric description of the final state. Eastern-European Journal of Enterprise Technologies, 3 (4 (87)), 51-63. doi: https://doi.org/10.15587/ 1729-4061.2017.105294

[14] Dymko, I. (2018). Choice of the optimal control strategy for the duplex-process of induction melting of constructional iron. EUREKA: Physics and Engineering, 4, 3-13. doi: https://doi.org/10.21303/2461-4262.2018.00669

[15] Makarenko, D. (2017). Investigation of the response surfaces describing the mathematical model of the influence of temperature and $\mathrm{BeO}$ content in the composite materials on the yield and ultimate strength. Technology Audit and Production Reserves, 3 (3 (35)), 13-17. doi: https://doi.org/10.15587/2312-8372.2017.104895

[16] Demin, D. (2018). Investigation of structural cast iron hardness for castings of automobile industry on the basis of construction and analysis of regression equation in the factor space «carbon (C) - carbon equivalent (Ceq)». Technology Audit and Production Reserves, 3 (1 (41)), 29-36. doi: https://doi.org/10.15587/2312-8372.2018.109097

[17] Demin, D. (2017). Synthesis of nomogram for the calculation of suboptimal chemical composition of the structural cast iron on the basis of the parametric description of the ultimate strength response surface. ScienceRise, 8 (37), 36-45. doi: https:// doi.org/10.15587/2313-8416.2017.109175

[18] Domina, O., Lunin, D., Barabash, O., Balynska, O., Paida, Y., Mikhailova, L., Niskhodovska, O. (2018). Algorithm for selecting the winning strategies in the processes of managing the state of the system «supplier - consumer» in the presence of aggressive competitor. Eastern-European Journal of Enterprise Technologies, 6 (3 (96)), 48-61. doi: https://oi.org/10.15587/ $1729-4061.2018 .152793$ 